\title{
A vortex-based model for the subgrid flux of a passive scalar
}

\author{
D. I. Pullin \\ Graduate Aeronautical Laboratories 105-50, California Institute of Technology, Pasadena, California 91125
}

(Received 29 September 1999; accepted 1 June 2000)

\begin{abstract}
A model for the flux of a passive scalar by the subgrid motions in the large-eddy simulation of turbulent flow is proposed within the framework of the stretched-vortex subgrid stress model. The model is based on an analytical solution for the winding of a scalar field by an elemental subgrid vortex. This gives a tensor gradient-diffusion expression for the local flux of the scalar with subgrid turbulent diffusivity which depends upon the subgrid energy, the local cell size, and the vortex orientation in space. The scalar-flux subgrid model is tested by comparison of the results of $32^{3}$ large-eddy simulation of passive-scalar transport by forced isotropic turbulence in the presence of a mean scalar gradient, with the direct-numerical simulation results of Overholt and Pope [Phys. Fluids 8, 2128 (1996)]. The present large-eddy simulation results predict that at large TaylorReynolds numbers, the ratio of the scalar variance to the squared product of the scalar gradient with the dissipation length of the turbulence, is asymptotic to a nearly constant value $c^{\prime 2} /\left(\alpha_{1} L_{\epsilon}\right)^{2}$ $\approx 0.36$. (C) 2000 American Institute of Physics. [S1070-6631(00)00809-6]
\end{abstract}

\section{INTRODUCTION}

The mixing of a passive scalar by a rapidly fluctuating flow field is a classical problem in turbulence with many applications, including multiphase flows, environmental flows involving the dispersion of both active and passive scalars, and turbulent combustion. The classical scaling laws (see Tennekes and Lumley, ${ }^{1}$ Chap. 8) give insight into the physics of mixing but do not provide a general means of calculating the scalar statistics in a general turbulent flow. One promising and increasing popular approach to the estimation of turbulence characteristics for a wide variety of flows is the use of large-eddy simulation (LES). The main idea is to directly evolve those large turbulent scales (resolved flow) that are expected to show strong dependence on the initial and boundary conditions driving the flow, while modeling the residual stresses produced by the subgrid (unresolved) scales, which might be supposed to exhibit general characteristics described by a few parameters dependent mainly on the local flow structure near the resolved-flow cutoff. For many turbulence applications the transport mechanisms for mass, momentum, and energy are often dominated by the large, resolved scales. This may not be true for the transport of a passive scalar, where, at large Schmidt numbers, transport at sub-Kolmogorov scales may be important.

The last decade has seen a great deal of interest in both the development of subgrid-stress (SGS) models for the LES of turbulent flows (see Lesieur and Métais ${ }^{2}$ for a recent review) and in the analysis of the performance of these models using different numerical schemes for the computation of turbulent flows. One means of incorporating passive scalar transport into LES has been to introduce the idea of a turbulent Prandtl number $\operatorname{Pr}_{t}$ into the LES methodology, combined with use of a simple gradient approximation to the flux terms that arise when small-scale filtering is applied to the scalar convection-diffusion equation. Closure is obtained when either $\operatorname{Pr}_{t}$ is specified as a model parameter or is estimated using a dynamic procedure. ${ }^{3}$

In the present paper we follow a different approach by extending the stretched-vortex SGS model for LES to include the transport of a passive scalar by turbulent flows. The residual term in the filtered convection-diffusion equation for the scalar $c$ is modeled by analysis of the distortion of the local resolved scalar field by an assumed axisymmetric subgrid vortex. This gives an expression for the subgrid scalar flux in terms of the scalar gradients and locally known properties of the stretched-vortex SGS model. In Sec. II the modeling problem is defined and a brief review of the stretchedvortex SGS model for the filtered momentum equations is given. This is followed in Sec. III by an account of the present subgrid scalar (SGSc) model. In Sec. IV we describe the application of the model to the LES of passive scalar stiring by forced homogeneous turbulence in the presence of a mean scalar gradient. The results including comparison with $\mathrm{DNS}^{4}$ are discussed in Sec. V.

\section{EQUATIONS FOR LARGE-EDDY SIMULATION}

\section{A. LES equations}

We describe a model for the subgrid flux of a passive scalar based on the stretched-vortex model. Our treatment is restricted to a passive scalar but is easily extended to an active scalar, for example, in variable density stratified flows. The reader interested mainly in results of the present LES rather than the model details can skip to Sec. IV. We consider the LES of the Navier-Stokes equations on a grid with a typical cell size $\Delta$ and time step $\Delta t$. The LES equations are

$$
\frac{\partial \widetilde{U}_{i}}{\partial x_{i}}=0
$$




$$
\frac{\partial \widetilde{U}_{i}}{\partial t}+\frac{\partial}{\partial x_{j}}\left(\widetilde{U}_{i} \widetilde{U}_{j}\right)=-\frac{\partial \widetilde{P}}{\partial x_{i}}-\frac{\partial T_{i j}}{\partial x_{j}}+\nu \frac{\partial^{2} \widetilde{U}_{i}}{\partial x_{j} \partial x_{j}}+F_{i},
$$

where, $T_{i j}$ represents the effect of subgrid dynamics, $F_{i}$ is a forcing term, and the resolved fields are defined by a tilde. The above-mentioned equations are not closed and require a scheme or algorithm for computing $T_{i j}$ from $\widetilde{U}_{i}$. This is the SGS model. For incompressible constant density flow, the equivalent equation for a passive scalar $c$ is

$$
\frac{\partial \tilde{c}}{\partial t}+\frac{\partial}{\partial x_{j}}\left(\tilde{c} \widetilde{U}_{j}\right)=-\frac{\partial g_{j}}{\partial x_{j}}+D \frac{\partial^{2} \tilde{c}}{\partial x_{j} \partial x_{j}},
$$

where $g_{j}$ can be interpreted as the subgrid flux of $c$ by the turbulent velocity field and $D$ is the molecular diffusivity. The above equations may be obtained by applying filtering techniques to the Navier-Stokes and scalar convectiondiffusion equations. ${ }^{2,3}$

\section{B. The stretched-vortex SGS model}

The stretched-vortex SGS model ${ }^{5}$ was developed from application of vortex-based models to calculate properties of the fine scales of turbulence. ${ }^{6}$ It has been argued $^{5}$ that the volume-averaged stresses produced by an ensemble of (noninteracting) straight vortices can be expressed in terms of the subgrid energy contained in the range $k>k_{c}$ as

$$
T_{i j}=2 \int_{k_{c}}^{\infty} E(k) d k\left\langle E_{p i} Z_{p q} E_{q j}\right\rangle .
$$

In (3), $E(k)$ is the subgrid energy spectrum, $E_{i j}$ is the rotation matrix for transformation from vortex-fixed to laboratory axes, $Z_{i j}$ is a diagonal tensor with diagonal elements $\left(\frac{1}{2}, \frac{1}{2}, 0\right),\left\langle E_{p i} Z_{p q} E_{q j}\right\rangle$ is a rotation from vortex-fixed to laboratory axes averaged over the probability distribution function (pdf) $P(\alpha, \beta)$ of the Euler angles $\alpha, \beta$ describing the orientation of the subgrid vortex axis relative to laboratory axes, and $k_{c}=\pi / \Delta$ is a cutoff wave number. Use of (3) as the basis of a viable SGS model requires knowledge of both $E(k)$ and $P$ in terms of the resolved field.

Several simple axis-orientation models based on deltafunctions models of $P(\alpha, \beta)$ within cells have been proposed and tested. ${ }^{7,8}$ These include models based on alignment of the vortex axis with the eigenvectors corresponding to the maximum and intermediate eigenvalues of the resolved rateof-strain tensor $\widetilde{S}_{i j}$ (eigenvector-alignment model), alignment with the resolved vorticity (vorticity-alignment model), and a model in which the alignment vector was calculated dynamically using an evolution equation which describes the tilting and rotation of the vortex axis by the local resolved velocity gradient field (rotation model). For delta-function $P(\alpha, \beta)$ the subgrid stresses are

$$
T_{i j}=\left(\delta_{i j}-e_{i}^{v} e_{j}^{v}\right) K, \quad K=\int_{k_{c}}^{\infty} E(k) d k,
$$

where $K$ is the subgrid energy and $e_{i}^{v}$ are the direction cosines of the vortex axis. In the LES runs to be discussed subsequently, the subgrid-vortex eigenvector alignment model denoted as Model 1a (Misra and Pullin ${ }^{7}$ ) was used. For estimation of $K$ we consider two models for $E(k)$.

\section{Sharp viscous cutoff}

The first of these models assumes a simple Kolmogorov form of $E(k)$, with a viscous cutoff

$$
E(k)=\left\{\begin{array}{l}
\mathcal{K}_{0} \epsilon^{2 / 3} k^{-5 / 3}, \quad k_{c}<k<J \eta^{-1}, \\
0, \quad k>J \eta^{-1},
\end{array}\right.
$$

where $\mathcal{K}_{0}$ is the Kolmogorov prefactor, $\eta=\left(\nu^{3} / \epsilon\right)^{1 / 4}$ is the local Kolmogorov length, $\epsilon$ being the local dissipation, and $J$ is a cutoff parameter. To make use of (5) requires knowledge of $\mathcal{K}_{0} \epsilon^{2 / 3}$. In the present simulations this is obtained dynamically using a physical space version of the present model $^{8}$ based on the relationship between $E(k)$ and the second-order velocity structure function $\widetilde{F}_{2}(r)$ $=\overline{\delta U_{1}^{2}(\mathbf{r})+\delta U_{2}^{2}(\mathbf{r})+\delta U_{3}^{2}(\mathbf{r})}$ in physical space, where the overbar refers to some suitable average and $\delta$ refers to a difference at separation $r$. The main idea ${ }^{9}$ is to calculate $\widetilde{F}_{2}$ at scales near the resolved scale cutoff using the kinematics of the particular SGS model. Values of $\widetilde{F}_{2}$ are estimated by averaging the local resolved field over either a circle (fourpoint average) or sphere (six-point average) centered on the cell center with radius $r=\Delta$, and these are then used to estimate the product $\mathcal{K}_{0} \epsilon^{2 / 3}$ in the assumed subgrid energy spectrum. For the homogeneous "box" turbulence of the present LES we use a spherical average at $r=\Delta$ (although a circular average, used for near wall flows, works just as well $^{8}$ ) for which it can be shown, using (5), that ${ }^{8,9}$

$$
\begin{aligned}
& \mathcal{K}_{0} \epsilon^{2 / 3}=\frac{\widetilde{F}_{2}}{A \Delta^{2 / 3}} \\
& A=4 \int_{0}^{\pi} u^{-5 / 3}\left(1-\frac{\sin u}{u}\right) d u \approx 1.90695 \\
& \widetilde{F}_{2}(\Delta)=\frac{1}{6} \sum_{j=1}^{6}\left(\delta \widetilde{U}_{1}^{2}+\delta \widetilde{U}_{2}^{2}+\delta \widetilde{U}_{3}^{2}\right)_{j} .
\end{aligned}
$$

The right-hand side of (7) is a local estimate of $\widetilde{F}_{2}$ using a six-point stencil. Once $\mathcal{K}_{0} \epsilon^{2 / 3}$ is known, $K$ follows from (4)(5) as

$$
K=\left\{\begin{array}{l}
\frac{3 \mathcal{K}_{0} \epsilon^{2 / 3}}{2 k_{c}^{2 / 3}}\left[1-\left(\frac{k_{c} \eta}{J}\right)^{2 / 3}\right], \quad k_{c}<J \eta^{-1}, \\
0, \quad k_{c}>J \eta^{-1} .
\end{array}\right.
$$

When it is assumed that $J \rightarrow \infty, K$ can be calculated immediately and $T_{i j}$ follows from (4). For finite $J$, the righthand side of (8) cannot be evaluated because although the group $\mathcal{K}_{0} \epsilon^{2 / 3}$ is known, $\epsilon$ itself, and therefore $\eta$, is not known. When a balance is assumed between the total local dissipation $\epsilon$ and the sum of the resolved-scale dissipation and the rate of transfer of turbulent kinetic energy from resolved to subgrid scales $\epsilon_{\mathrm{SGS}}=-T_{i j} \widetilde{S}_{i j}$, a single equation in each cell results with unknown $k_{c} \eta$. For specified $J$ this can be solved by Newton's method. $K$ then follows from (8) and local values of $\epsilon$ and $\mathcal{K}_{0}$ can be computed if required. Full 
details are given in Sec. B4 of Ref. 8. A cutoff $k_{c} \eta=1(J$ $=1$ ) is preferred on the grounds that the energy spectrum is expected to decay very rapidly near the Kolmogorov scale.

\section{A spiral-vortex model}

As an alternative to the generic Kolmogorov-type spectrum (5) we can assume that the subgrid vortices are specifically of the stetched-spiral type proposed by Lundgren ${ }^{10}$ as a model for fine-scale turbulence, with energy spectrum of the form

$$
E(k)=\mathcal{K}_{0} \epsilon^{2 / 3} k^{-5 / 3} \exp \left(-2 k^{2} \nu /(3 a)\right),
$$

where $a$ is the external axial rate of strain. We adapt (10) as a subgrid model by replacing it with

$$
E(k)=\mathcal{K}_{0} \epsilon^{2 / 3} k^{-5 / 3} \exp \left(-2 k^{2} \nu /\left(3\left|S_{3}^{\prime}\right|\right)\right),
$$

where $S_{3}^{\prime}=\widetilde{S}_{i j} e_{i}^{v} e_{j}^{v}$ is the axial strain along the subgrid vortex axis provided by the local resolved flow. We ignore that $\widetilde{S}_{i j}$ is not axisymetric, as was assumed in the derivation ${ }^{10}$ of (10). The subgrid energy is then

$$
\begin{aligned}
& K=\mathcal{K}_{0} \epsilon^{2 / 3}\left(\frac{\nu}{12\left|S_{3}^{\prime}\right|}\right)^{1 / 3} \Gamma\left[-\frac{1}{3}, \frac{2 k_{c}^{2} \nu}{3\left|S_{3}^{\prime}\right|}\right], \\
& \Gamma[a, Y]=\int_{Y}^{\infty} t^{a-1} e^{-t} d t,
\end{aligned}
$$

where $\Gamma[a, Y]$ is the incomplete gamma function. In order to estimate $\mathcal{K}_{0} \epsilon^{2 / 3}$ we use a large Reynolds number approximation and put $\nu=0$ in (12), allowing use of (6) and (7). We remark that since $S_{3}^{\prime}$ is available from the resolved field and the vortex orientation model, (12) is free of parameters, save for $k_{c}$. Fast evaluation of $\Gamma[-1 / 3, Y]$ accurate to three figures (sufficient for LES) is straightforward using a four-point table lookup in $0.4<Y<4$ combined with asymptotic expressions for $Y$ outside this range.

\section{SUBGRID MODEL FOR THE FLUX OF A PASSIVE SCALAR}

\section{A. Convection of a scalar by an axisymmetric vortex}

We model $g_{j}$ of Eq. (2) by an analytical treatment of the convection of the $c$ field by the axisymmetric model of a subgrid vortex. This is an extremely simplified description of the scalar transport by a vortical flow but one which it is hoped will capture sufficient of the subgrid transport physics to give a reasonable averaged representation of the subgrid flux. We work in subgrid-vortex polar coordinates $\left(r, \theta, x_{3}^{\prime}\right)$ with $x_{3}^{\prime}$ along the vortex axis. The subgrid velocity field is assumed to be

$$
u_{\theta}=r \Omega(r), \quad u_{r}=u_{x_{3}^{\prime}}=0,
$$

where $\Omega(r)$ is the local angular velocity. The scalar convection equation is then

$$
\frac{\partial c}{\partial t}+\Omega(r) \frac{\partial c}{\partial \theta}=0,
$$

where we have neglected the effect of scalar diffusion compared to convection within the vortex over a typical LES time step. We expect this to be a physically reasonable assumption provided that the scalar diffusion coefficient $D$ is at most of order the molecular viscosity $\nu$; it will be argued subsequently that the present model is appropriate for Schmidt numbers $\mathrm{S}_{\mathrm{c}}=O(1)$.

The solution of (14) is taken to be of the form

$$
\begin{aligned}
c\left(r, \theta, x_{3}^{\prime}, t\right)= & \sum_{n=-\infty}^{\infty} \sum_{m=-\infty}^{\infty} c_{n m}(r) e^{i n(\theta-\Omega t)+i \pi m x_{3}^{\prime} / L} \\
& +\frac{\partial \tilde{c}}{\partial x_{1}^{\prime}} r \cos (\theta-\Omega t)+\frac{\partial \tilde{c}}{\partial x_{2}^{\prime}} r \sin (\theta-\Omega t) \\
& +\frac{\partial \tilde{c}}{\partial x_{3}^{\prime}} x_{3}^{\prime}, \quad c_{-n,-m}=c_{n m}^{*},
\end{aligned}
$$

where primed Cartesian variables refer to vortex-fixed coordinates $\left(x_{1}^{\prime}, x_{2}^{\prime}, x_{3}^{\prime}\right)$ with $x_{1}^{\prime}=r \cos \theta, x_{2}^{\prime}=r \sin \theta$ lying in a plane normal to the vortex axis. In (15), $\partial \tilde{c} / \partial x_{j}^{\prime}$ is the local gradient of the resolved scalar field in vortex coordinates and $c_{n m}(r)=a_{n m} e^{i \psi_{n m}}$ are the Fourier coefficients of the subgrid background scalar field, where $a_{n m} \geqslant 0$ are real amplitudes and $\psi_{n m}$ are phase angles. The initial condition, obtained by putting $t=0$ in (15), is comprised of the sum of the background field plus components given by the leading terms in the Taylor expansion of the local resolved field, with coefficients $\partial \tilde{c} / \partial x_{j}^{\prime}$.

We model $g_{j}^{\prime}$ as the flux of $c$ by the velocity field of the vortex in a time interval $0 \leqslant t \leqslant T$ ( $T$ is a mixing time) by taking a volume-time average of the product $u_{j}^{\prime} c$ inside a cylinder of length $2 L$ and radius $R_{1}$ whose axis is coincident with that of the vortex. This can be written as

$$
\begin{aligned}
g_{1}^{\prime}+i g_{2}^{\prime}= & \frac{1}{2 \pi R_{1}^{2} L T} \int_{-L}^{L} \int_{0}^{2 \pi} \int_{0}^{R_{1}} \int_{0}^{T} c\left(r, \theta, x_{3}^{\prime}, t\right) \\
& \times i u_{\theta} e^{i \theta} d x_{3}^{\prime} d \theta r d r d t,
\end{aligned}
$$

together with $g_{3}^{\prime}=0$. Substituting (13) and (15) into (16) and performing the integrals with respect to $x_{3}^{\prime}, \theta$, and $t$ then gives

$$
\begin{gathered}
g_{1}^{\prime}+i g_{2}^{\prime}=\frac{1}{R_{1}^{2} T} \int_{0}^{R_{1}} r^{2}\left(e^{i \Omega T}-1\right)\left(2 c_{-1,0}(r)+r \mathcal{C}\right) d r \\
\mathcal{C}=\frac{\partial \tilde{c}}{\partial x_{1}^{\prime}}+i \frac{\partial \tilde{c}}{\partial x_{2}^{\prime}} .
\end{gathered}
$$

Next we now introduce the vortex circulation scale $\Gamma$ and radius $R \leqslant R_{1}$ such that

$$
\Omega(r)=\frac{\Gamma}{R^{2}} \Omega(\xi), \quad \xi=\frac{r}{R}, \quad \sigma=\frac{\Gamma T}{R^{2}},
$$

where $\Omega(\xi)$ is the dimensionless angular velocity and $\sigma$ is the dimensionless circulation scale. We make a random phase approximation for the coefficient $c_{-1,0}(r)$ and average (17) over the phase angle $\psi_{-1,0}$ and also over the pdf of $\Gamma$ assumed symmetrical about $\Gamma=0$ (i.e., $\Gamma=0$ is equally likely to be positive/negative), to obtain 


$$
g_{1}^{\prime}+i g_{2}^{\prime}=\frac{R^{4}}{R_{1}^{2} T} \mathcal{C} \int_{0}^{R_{1} / R} \xi^{3}[\cos (\sigma \Omega(\xi))-1] d \xi .
$$

The kinetic energy $K_{v}$ per unit mass due to the velocity field of the vortex contained within the cylinder of radius $R_{1}$ can written as

$$
K_{v}=\frac{\Gamma^{2}}{R_{1}^{2}} \int_{0}^{R_{1} / R} \xi^{3} \Omega^{2}(\xi) d \xi .
$$

Scaling arguments can be used to estimate $\sigma=O(1)$. The main contribution to the integral in (19) can then be expected to come from $\xi=O(1)$, where $\Omega \ll 1$. The integral can then be approximated by retaining only the leading terms in a Taylor series expansion of the cosine to give

$$
g_{1}^{\prime}+i g_{2}^{\prime} \approx-\frac{1}{2} \frac{\Gamma^{2} T}{R_{1}^{2}} \mathcal{C} \int_{0}^{R_{1} / R} \xi^{3} \Omega(\xi) d \xi .
$$

The numerical accuracy of this approximation was tested for a class of functions $\Omega(\xi)$ used to model the fine scale properties of isotropic turbulence. ${ }^{6}$ Errors were found to be uniformly small when $\sigma=O(1)$ or smaller. Comparing (21) with (20) then gives

$$
g_{j}^{\prime}=-\frac{1}{2} K_{v} T\left(\frac{\partial \tilde{c}}{\partial x_{j}^{\prime}}\right), \quad j=1,2 .
$$

The dependence of $g_{j}^{\prime}$ on the vortex variables $R, \Gamma$, the volume dimension $R_{1}$, and the vortex velocity $r \Omega$ is now contained entirely in the vortex energy $K_{v}$. The physical content of (22) is that the main contribution to the subgrid scalar transport is produced by the distortion of the filtered (at the cell level) scalar gradient by the subgrid motion. The model states the intuitively reasonable result that the subgrid scalar flux is proportional to the product of the subgrid energy with a characteristic mixing time. The preceding analysis does not include the possible effects of vortex nonaxisymmetry on the subgrid scalar flux. A detailed calculation based on use of the velocity field of the stretched-spiral vortex ${ }^{10}$ in place of (13) shows that nonaxisymmetry does not contribute to the scalar flux produced by the winding of the local resolved field after an average is taken over the angle of spin of the spiral structure about its axis, when this angle is assumed to be distributed uniformly on the unit circle. This requires replacement of (15) with a solution of the analog of (14) for the spiral vortex, together with appropriate averaging. The details are lengthy and are omitted presently.

\section{B. A subgrid scalar-flux model}

Transforming to laboratory coordinates $x_{i}$, (22) can be written as

$$
g_{j}=-\mu_{j p} \frac{\partial \tilde{c}}{\partial x_{p}}, \quad \mu_{j p}=\frac{1}{2} K_{v} T\left(\delta_{j p}-e_{j}^{v} e_{p}^{v}\right) .
$$

Equation (23) gives a tensor-eddy diffusivity model of the subgrid scalar flux. This form arises owing to the anisotropic character of the subgrid mixing, which is restricted to the plane normal to the vortex axis. This also gives the property that the model subgrid scalar-gradient produced by the transport is orthogonal to the subgrid vorticity, as represented by the vortex. This is consistent with the observations of Ruetsch and Ferziger, ${ }^{11}$ who found that for DNS fields, the probability density of the alignment between the vorticity and scalar gradient vectors shows a maximum when these vectors are orthogonal. The vortex energy $K_{v}$ is now identified with the subgrid energy $K$ as defined in (4), $K_{v} \equiv K$. Both $K$ and $e_{i}^{v}$ are available from the SGS model, but for closure, an estimate of $T$ is required. We assume presently that $T$ is a typical eddy turnover time for the subgrid motions, which we will approximate as $T=\gamma \Delta / K^{1 / 2}$, where $\gamma$ is a dimensionless constant. We now have a realizable model

$$
g_{j}=-\frac{\gamma \pi}{2 k_{c}} K^{1 / 2}\left(\delta_{j p}-e_{j}^{v} e_{p}^{v}\right) \frac{\partial \tilde{c}}{\partial x_{p}} .
$$

The local scalar dissipation, or more precisely, the rate of transfer of scalar variance from resolved to subgrid scales, is

$$
\epsilon_{\mathrm{SG}}^{c} \equiv-g_{j} \frac{\partial \tilde{c}}{\partial x_{j}}=\frac{\gamma \pi}{2 k_{c}} K^{1 / 2}\left(\delta_{j p}-e_{j}^{v} e_{p}^{v}\right) \frac{\partial \tilde{c}}{\partial x_{p}} \frac{\partial \tilde{c}}{\partial x_{j}} .
$$

Alignment of laboratory and vortex axes shows that $\epsilon_{\mathrm{SG}}^{c}$ $\geqslant 0$.

We can estimate $\gamma$ by an argument similar to that used by Lilly ${ }^{12}$ to estimate the Smagorinsky constant. First, (25) is averaged over all possible directions of the vortex axes that are assumed uniformly distributed on the unit sphere (equivalent to the assumption of locally isotropic turbulence), with both $K$ and $\partial \tilde{c} / \partial x_{j}$ held constant, to give

$$
\epsilon_{\mathrm{SG}}^{c}=\frac{\gamma \pi}{3 k_{c}} K^{1 / 2} \frac{\partial \tilde{c}}{\partial x_{j}} \frac{\partial \tilde{c}}{\partial x_{j}} .
$$

We remark that (26) resembles the dissipation produced by a gradient-diffusion model. Next an approximation for the square of the resolved scalar gradient at the cutoff scale $k_{c}$ $=\pi / \Delta$ is made as

$$
\frac{\partial \tilde{c}}{\partial x_{j}} \frac{\partial \tilde{c}}{\partial x_{j}}=2 \int_{0}^{k_{c}} k^{2} E^{c}(k) d k,
$$

where $E^{c}(k)$ is the scalar spectrum. The integral in (27) is evaluated using a Obukov-Corrsin spectrum of the form ${ }^{1}$ $E^{c}(k)=\beta \epsilon^{-1 / 3} \epsilon_{\mathrm{SG}}^{c} k^{-5 / 3}$ where $\beta$ is the Obukov-Corrsin prefactor. When this is done, $K$ is calculated from (9) (with $J \rightarrow \infty$ ), and with the results used in (26), it is found that

$$
\gamma=\frac{2}{\pi}\left(\frac{2}{3 \mathcal{K}_{0}}\right)^{1 / 2} \frac{1}{\beta} .
$$

Typical values of $\mathcal{K}_{0}=1.5-2.0$ and $\beta=0.4-0.5$ then give $\gamma$ in the range $0.74-1.1$. If (27) is replaced by a differenced approximation to the square of the scalar gradient based on the second-order scalar structure function at separation $r$ $=\Delta$ [the analysis is analogous to that giving (7)], one can obtain

$$
\gamma=\frac{\pi^{1 / 3}}{A}\left(\frac{2}{3 \mathcal{K}_{0}}\right)^{1 / 2} \frac{1}{\beta} .
$$


This gives $\gamma=0.89-1.3$ for the above range of $\mathcal{K}_{0}, \beta$. All present calculations were done with $\gamma=1.0$.

We remark that for the sharp viscous cutoff model of the subgrid $E(k)$, the SGS model will turn off (smoothly) when $k_{c} \eta=J$, for which $K \rightarrow 0$. From (24) it follows that $g_{j} \rightarrow 0$. This is reasonable physically for Schmidt numbers $\nu / D$ $=O(1)$. For $\nu / D \gg 1$ the present model may still provide a useful representation of the scalar transfer to the viscousconvective subrange provided that a good estimate of $K$ is available. This may require a more refined model of the subgrid $E(k)$ in the far viscous range than those used presently and will be left for future work; the present test of the scalar subgrid model will be restricted to $\mathrm{Sc}=0.7$.

\section{PASSIVE-SCALAR MIXING BY FORCED HOMOGENEOUS TURBULENCE}

\section{A. Scalar field with a mean gradient}

We test the present subgrid model using LES of passivescalar transport by a forced turbulent flow field in the presence of a mean scalar gradient. The turbulence is spatially periodic in a $(2 \pi)^{3}$ box. To obtain an equation for the resolved scalar fluctuation, we put

$$
\tilde{c}=\alpha_{j} x_{j}+c^{\prime},
$$

in (2) where $\alpha_{j}$ is the mean scalar gradient and $c^{\prime}(\mathbf{x}, t)$ is the resolved scalar fluctuation about the mean. Using (30) and taking the gradient of (2), integrating over the $(2 \pi)^{3}$ volume and assuming that all fluctuating quantities are spatially periodic then shows that $\alpha_{i}$ is preserved by the evolution. ${ }^{4} \mathrm{An}$ equation for the resolved scalar variance $\left(c^{\prime}\right)^{2}$ for spatially periodic fields is given by

$$
\frac{1}{2} \frac{\partial}{\partial t}\left\langle c^{\prime 2}\right\rangle+\alpha_{j}\left\langle c^{\prime} \widetilde{U}_{j}\right\rangle=\left\langle g_{j} \frac{\partial c^{\prime}}{\partial x_{j}}\right\rangle-D\left\langle\left(\frac{\partial c^{\prime}}{\partial x_{j}}\right)^{2}\right\rangle,
$$

where \langle\rangle is a volume average at fixed time. The second term on the left-hand side of (31) is the production of scalar variance by the scalar flux working against the mean scalar gradient. On the right-hand side the first term is the volume average of the subgrid scalar dissipation (transfer off the grid) while the second term is the molecular dissipation.

Overholt and Pope, ${ }^{4}$ henceforth referred to as OP, conducted DNS of the mixing of a passive scalar in the presence of a mean scalar gradient in one direction, by forced, spatially periodic homogeneous turbulence. In this flow a statistical steady-state-equilibrium scalar variance is achieved owing to the balance of scalar variance production and scalar dissipation. OP performed DNS studies at $\mathrm{Re}_{\lambda} \approx 28-180$ at resolutions in the range $32^{3}-256^{3}$ from which they obtained time-volume-ensemble averaged statisics over several independent simulations at each $\operatorname{Re}_{\lambda}$. Presently, we test the scalar-flux/stretched-vortex model subgrid model by LES of scalar transport at conditions similar to OP.

We solved (1) and (2) with forcing $F_{i}$, using periodic boundary conditions in all three directions, and implemented the stretched-vortex SGS model and scalar subgrid model described previously. A Fourier-Galerkin pseudospectral method $^{7}$ was used with $3 / 2$ dealiasing for the nonlinear terms both in the momentum and scalar equations. The resolution of all present LES is $32^{3}$ so that the nonlinear terms were treated using $48^{3}$ modes. A second-order explicit RungeKutta scheme was used for time advancement after first writing the spectral equations in an integrating-factor form. Forcing was implemented by exciting low wave numbers such that the total energy injection rate remained constant in time. Fourier modes were forced within a wave number shell $|\mathbf{k}|$ $=k_{0}$ such that the energy injection rate, $\Sigma \hat{\mathbf{f}}_{k} \cdot \hat{\mathbf{U}}_{k}$, was constant and equal to $\varepsilon_{f}$. We used $k_{0}=2, N=20-$ a box of side 2 grid units centered around the origin with the center modes and the origin omitted. ${ }^{7}$

\section{B. LES runs performed}

Each LES was done by first initializing both the $\widetilde{U}_{i}$ and $c^{\prime}$ fields at dimensionless time $t=0$, using initial energy and $c^{\prime}$ spectra of the form $B k^{4} \exp \left(-\left(k / k_{r}\right)\right)^{2}$, where $B$ is an amplitude and $k_{r}$ a reference wave number, coupled with random values of the phase angles. Different values of $B$ and $k_{r}$ together with different seed values for the random number generator utilized in calculating the phases were used to produce statistically independent flow realizations. All LES runs used the same forcing parameter $\varepsilon_{f}=0.1$, with values of $\nu$ set to obtain desired statistical steady state values of the Taylor Reynolds number $\operatorname{Re}_{\lambda}=u^{\prime} \lambda / \nu$ and integral-length Reynolds number $\operatorname{Re}=u^{\prime} L / \nu$, where $\lambda$ is the Taylor microscale, $L$ is the integral length, and $u^{\prime}$ is the root-mean square velocity. All runs used $\alpha_{1}=1, \alpha_{2}=\alpha_{3}=0$. The large-eddy turnover time $t_{c}=L / u^{\prime}$ varied in the range 2.0-2.7 time units, depending on $\mathrm{Re}_{\lambda}$. Each LES was run for 20 dimensionless time units before taking statistics, which were obtained in a time window $20 \leqslant t \leqslant 80$. Calculations were done for nominal values of $\mathrm{Re}_{\lambda}=27-1540$. A Schmidt number $\mathrm{Sc}=\nu / D=0.7$ was used for all present DNS and LES runs.

At a fixed time, volume-averaged parameters were calculated as

$$
\begin{aligned}
u^{\prime 2}= & \frac{2}{3} \int_{0}^{k_{c}} E(k) d k+\frac{2}{3}\langle K\rangle, \\
\varepsilon= & \left\langle 2 \nu \widetilde{S}_{i j} \widetilde{S}_{i j}+\epsilon_{\mathrm{SGS}}\right\rangle, \\
\lambda^{2}= & \frac{15 \nu u^{\prime 2}}{\varepsilon}, \\
L= & \frac{\pi}{2 u^{\prime 2}}\left[\int_{0}^{k_{c} E(k)} \frac{u^{2}}{k} d k\right. \\
& \left.+\left\langle\frac{3 \mathcal{K}_{0} \epsilon^{2 / 3} k_{c}^{-5 / 3}}{5}\left(1-\left(\frac{k_{c} \eta}{J}\right)^{5 / 3}\right)\right\rangle\right], \\
L_{\epsilon}= & \frac{u^{\prime 3}}{\varepsilon}, \\
c^{\prime 2} \equiv & \left\langle\left(c-\alpha_{1} x_{1}\right)^{2}\right\rangle=2 \int_{0}^{k_{c}} E^{c}(k) d k+\left\langle c^{\prime \prime 2}\right\rangle, \\
\varepsilon^{c}= & \left\langle D\left(\frac{\partial c^{\prime}}{\partial x_{i}}\right)\left(\frac{\partial c^{\prime}}{\partial x_{i}}\right)+\epsilon_{\mathrm{SG}}^{c}\right),
\end{aligned}
$$


where $E(k)$ and $E^{c}(k)$ are the resolved-scale velocity (energy) and scalar spectrum, respectively, $\varepsilon$ and $\varepsilon^{c}$ are the volume-averaged dissipation of energy and scalar variance, respectively, $c^{\prime 2}$ is the scalar variance, and $L_{\epsilon}$ is the dissipation length. In each of (32), (33), (35), (37), and (38), the second term on the right-hand side gives the subgrid contribution to the defined quantity. An estimate of $c^{\prime \prime 2}$, the subgrid contribution to the scalar variance, was made by first assuming a subgrid scalar spectrum of the Obukov-Corrsin form (inertial-convective subrange)

$$
E_{\mathrm{SG}}^{c}(k)=\left\{\begin{array}{l}
\beta \epsilon^{-1 / 3} \epsilon^{c} k^{-5 / 3}, \quad k_{c}<k<J / \eta^{c}, \\
0, \quad k>J / \eta^{c},
\end{array}\right.
$$

where $\eta^{c}=\left(D^{3} / \epsilon\right)^{1 / 4}$. The prefactor is calculated using a sixpoint estimate of the scalar structure function $\widetilde{F}_{2}^{c}$

$$
\beta \epsilon^{-1 / 3} \epsilon^{c}=\frac{\widetilde{F}_{2}^{c}}{A \Delta^{2 / 3}}, \quad \widetilde{F}_{2}^{c}(\Delta)=\frac{1}{6} \sum_{j=1}^{6} \delta c^{\prime 2},
$$

where $A$ is defined in (7). An estimate of $c^{\prime \prime 2}$ is then given by

$$
c^{\prime \prime 2}=\left\{\begin{array}{l}
\frac{3 \beta \epsilon^{-1 / 3} \epsilon^{c}}{2 k_{c}^{2 / 3}}\left[1-\left(\frac{k_{c} \eta^{c}}{J}\right)^{2 / 3}\right], \quad k_{c}<J / \eta^{c}, \\
0, \quad k_{c}>J / \eta^{c} .
\end{array}\right.
$$

In (41) $\epsilon$ and $\epsilon^{c}$ refer to instantaneous local values of the energy and scalar dissipation respectively. Subsequently, except where otherwise specified, all LES results reported presently refer to volume-time-ensemble (VTE) averages over eight independent LES realizations at each $\operatorname{Re}_{\lambda}$.

When the sharp cutoff model (5) is used for the subgrid $E(k), J$ remains as a model parameter. Experience with this model indicates that $J=O(1)$ or larger has a quite small influence on the model performance for the decaying turbulence test at $\operatorname{Re}_{\lambda} \approx O(80){ }^{7,8}$ This may not be true at lower $\operatorname{Re}_{\lambda}$ or for the present subgrid scalar (SGSc) model and its performance in mixing by forced turbulence. We therefore performed LES runs with both SGS and SGSc models turned on at values of $J=1.0$ and $J=\infty$ in order to investigate the effect of $J$ on the variation of the turbulence and scalar statistics with $\operatorname{Re}_{\lambda}$. We refer to these as LES $(J=1.0)$ and LES $(J=\infty)$, respectively. Results obtained using (11) for the subgrid $E(k)$ will be referred to as LES(spiral). For these runs, $c^{\prime \prime 2}$ in (41) was evaluated with $J=\infty$.

\section{RESULTS AND DISCUSSION}

To test our code we performed DNS at $\operatorname{Re}_{\lambda} \approx 27$, for which both the SGS and SGSc models were turned off. Over 16 DNS runs our VTE averaged value of the scalar variance $c^{\prime 2} /\left(\alpha_{1} L_{\epsilon}\right)^{2}=0.9415$ lies just ouside the range $1.012 \pm 0.06$ found by OP, who also ensemble averaged over 16 DNS runs. It is possible that this may be due to the slightly different forcing used by us and OP, and to our use of dealiasing. Figure 1 depicts the time variation of $\operatorname{Re}_{\lambda}$ for particular LES realizations while Fig. 2 illustrates typical variations in the dimensionless scalar variance $c^{\prime 2} /\left(\alpha_{1} L_{\epsilon}\right)^{2}$ with $t$. For the $R_{\lambda}=180$ case in Fig. 2, the subgrid contribution $c^{\prime \prime 2}$ is always small compared with the resolved-scale scalar variance.

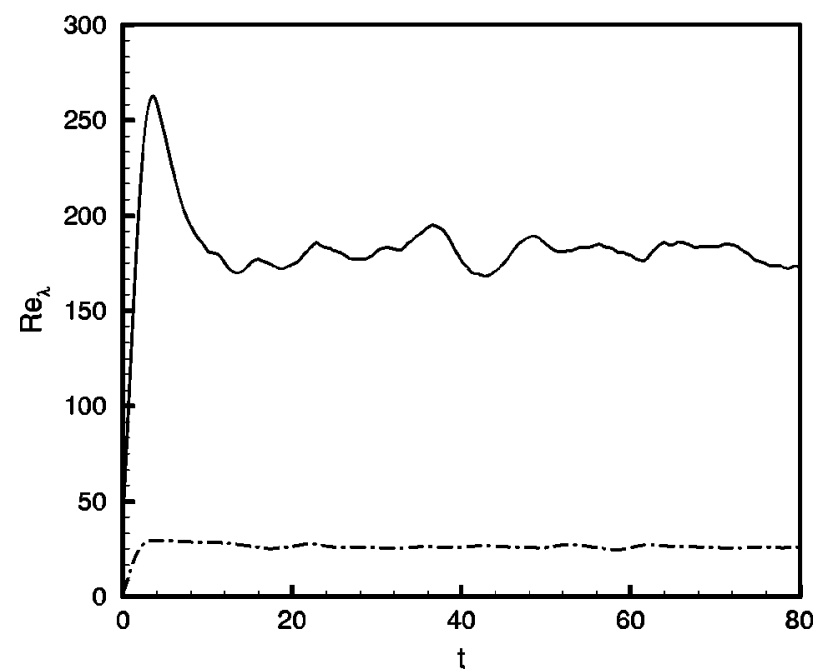

FIG. 1. Taylor Reynolds number $\operatorname{Re}_{\lambda}$ vs $t$. Dashed-dotted line: $\operatorname{Re}_{\lambda}=27$. Solid line: $\operatorname{Re}_{\lambda}=180$.

This was true of all present LES runs at $32^{3}$ resolution. In the present LES the cutoff scale $k_{c} \eta$ varied from 1.38 at $\operatorname{Re}_{\lambda}$ $\approx 27$ to $k_{c} \eta \approx 0.0006$ at $\operatorname{Re}_{\lambda} \approx 1540$. The LES is fully resolved only at our $\operatorname{Re}_{\lambda} \approx 27$, and is very considerably underresolved at our largest $\mathrm{Re}_{\lambda}$.

The main results of the present LES are displayed in Figs. 3 and 4, which show the variation of $c^{\prime 2} /\left(\alpha_{1} L_{\epsilon}\right)^{2}$ and $c^{\prime 2} /\left(\alpha_{1} L\right)^{2}$, respectively, versus $\operatorname{Re}_{\lambda}$. The general trend of the DNS of OP and the present LES are similar. For $\operatorname{Re}_{\lambda}$ $\leqslant 180$ the LES results are consistently below the DNS, but as pointed out previously our DNS values are below the OP values by a similar margin when both calculations are fully resolved and the SGS and SGSc models are quiescent. At $\operatorname{Re}_{\lambda} \approx 180$ the LES results for $c^{\prime 2} /\left(\alpha_{1} L_{\epsilon}\right)^{2}$ are higher than the OP value of 0.3052 . This was obtained from one $256^{3}$ run over about two large-eddy turnover times, and so may be subject to some statistical error, as of course may the present

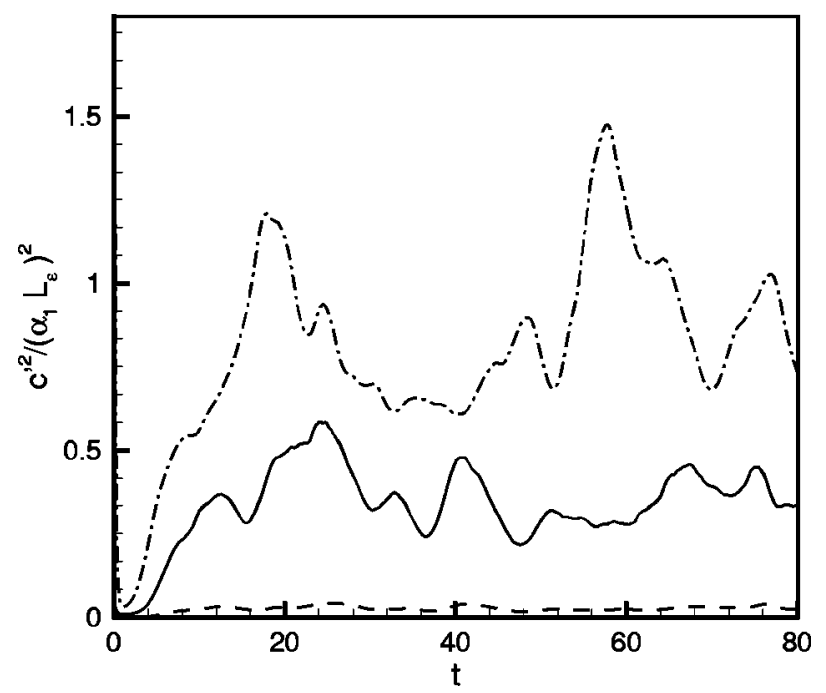

FIG. 2. Scalar variance $c^{\prime 2} /\left(\alpha_{1} L_{\epsilon}\right)^{2}$ vs $t$. Dashed-dotted line: $\operatorname{Re}_{\lambda}=27$. Solid line: resolved, Dashed line: subgrid, $\operatorname{Re}_{\lambda}=180$. 


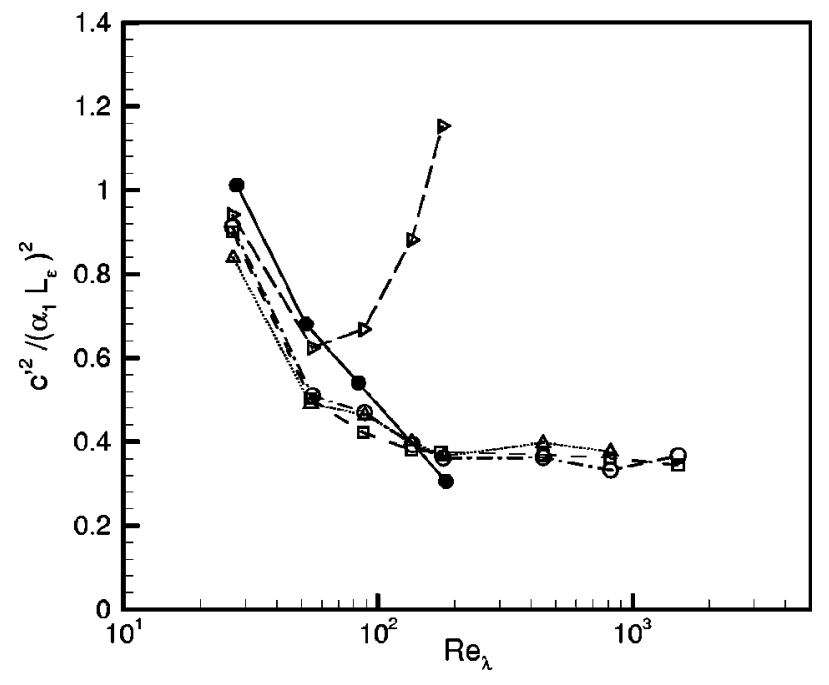

FIG. 3. Scalar variance $c^{\prime 2} /\left(\alpha_{1} L_{\epsilon}\right)^{2}$ vs $\operatorname{Re}_{\lambda}$. Closed circle: DNS (Ref. 4), square: LES (spiral), open circle: LES $(J=1.0)$, triangle: LES $(J=\infty)$, right triangle: LES with no scalar subgrid model.

LES results. When $\operatorname{Re}_{\lambda}$ is in the range 27-52, Figs. 3 and 4 show a moderate but definite effect of the subgrid model for $E(k)$ on the statistical steady state values, but when $\operatorname{Re}_{\lambda}$ $\geqslant 134$, this effect is within the statistical spread of the LES results. In particular the present LES predict that for $\operatorname{Re}_{\lambda}$ $\geqslant 180$ the scalar variance becomes essentially independent of $\operatorname{Re}_{\lambda}$ at near the constant values of $c^{\prime 2} /\left(\alpha_{1} L_{\epsilon}\right)^{2} \approx 0.36$ and $c^{\prime 2} /\left(\alpha_{1} L\right)^{2} \approx 1.50$. This independence of $\operatorname{Re}_{\lambda}$ is in agreement with the standard result from scaling arguments. ${ }^{1}$

Figure 5 shows the present LES results for the ratio of the time scales associated with the energy dissipation and the scalar dissipation

$$
r=\frac{3 u^{\prime 2} / \varepsilon}{c^{\prime 2} / \varepsilon^{c}}
$$

where $3 u^{\prime 2}$ is twice the turbulent kinetic energy. Both DNS and LES show an increase in $r$ with $\operatorname{Re}_{\lambda}$ but the LES indicates independence of $\operatorname{Re}_{\lambda}$ for $\operatorname{Re}_{\lambda}>180$ at a value near $r$

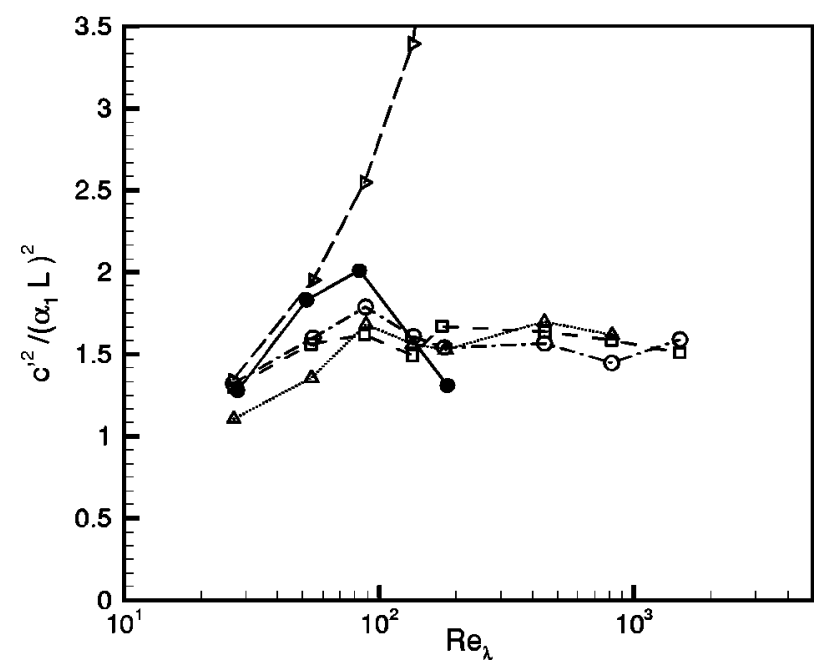

FIG. 4. Scalar variance $c^{\prime 2} /\left(\alpha_{1} L\right)^{2}$ vs $\operatorname{Re}_{\lambda}$. For key see Fig. 3.

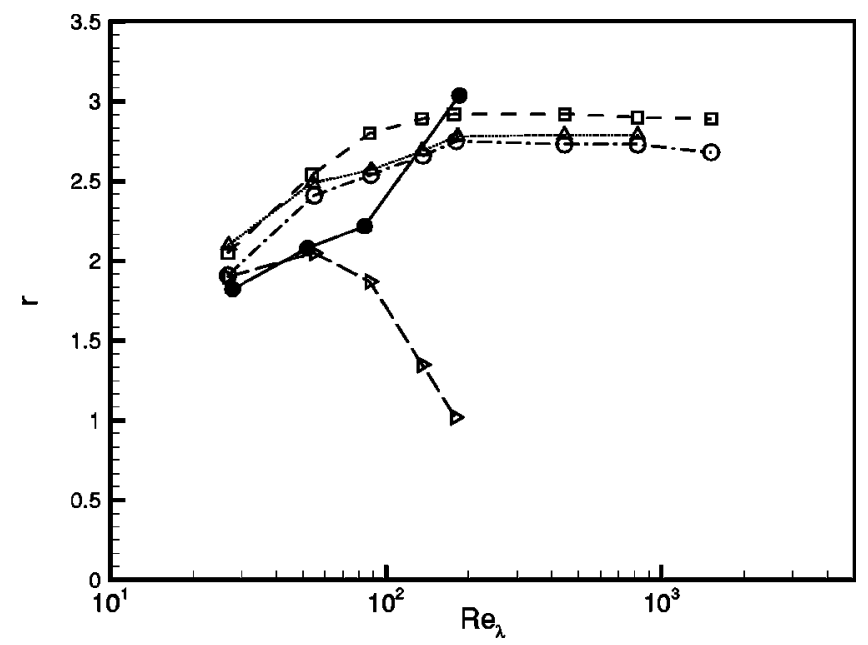

FIG. 5. Dissipation time ratio $r$ vs $\mathrm{Re}_{\lambda}$. For key see Fig. 3.

$\approx 2.8$. The use of constant $r$, independent of $\mathrm{Re}_{\lambda}$, is a well known modeling assumption. ${ }^{13}$ The present LES value lies outside the range of $0.7-2.4$ found by Warhaft and Lumley ${ }^{14}$ in reviewing heated-grid experiments. OP interpret their DNS results, and cite experimental results, as supporting the nonuniversal, flow-dependent character of this time-scale ratio. We remark that the forcing used in the LES may be another reason why $r$ is different than in natural or laboratory turbulence.

When the code was run with the SGS model turned on but the SGSc model turned off, i.e., $g_{j} \equiv 0$ in (2), it was found that the scalar simulation could run stably and achieve a statistical steady state. Results for these $\operatorname{LES}(J=1.0)$ runs (four realizations) with no SGSc model are displayed in Figs. $3-5$. These clearly demonstrate the large effect of the active SGSc model on the steady state scalar statistics. The effect of the SGSc model on the LES can be further seen in Fig. 6, which plots the ratio $\varepsilon_{\mathrm{SGS}}^{c} / \varepsilon^{c}$, of the subgrid scalar dissipation to the total scalar dissipation, versus $\mathrm{Re}_{\lambda}$. When $\operatorname{Re}_{\lambda}$

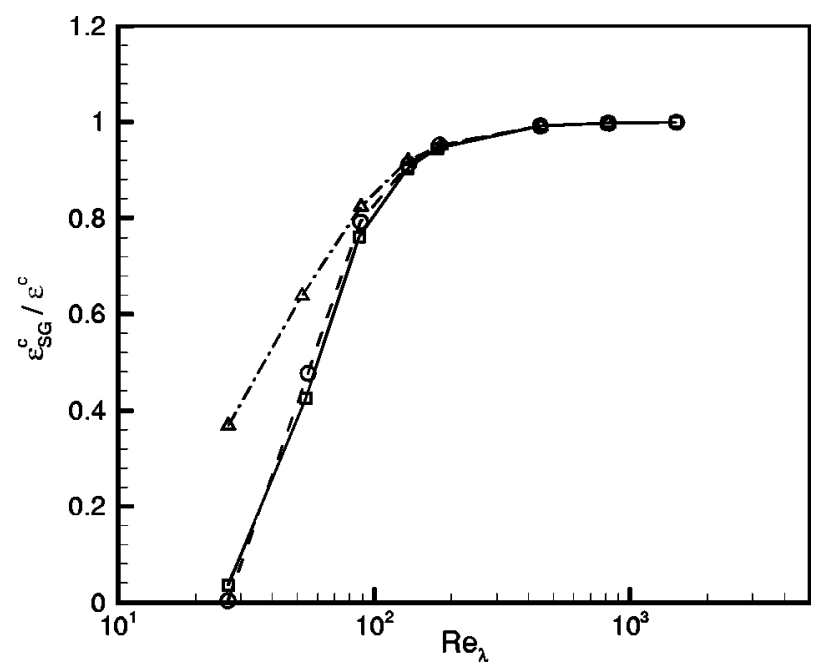

FIG. 6. Ratio $\varepsilon_{\mathrm{SGS}}^{c} / \varepsilon^{c}$ of subgrid scalar dissipation to total scalar dissipation vs $\operatorname{Re}_{\lambda}$. Square: LES (spiral), circle: LES $(J=1.0)$, triangle: LES $(J$ $=\infty)$. 


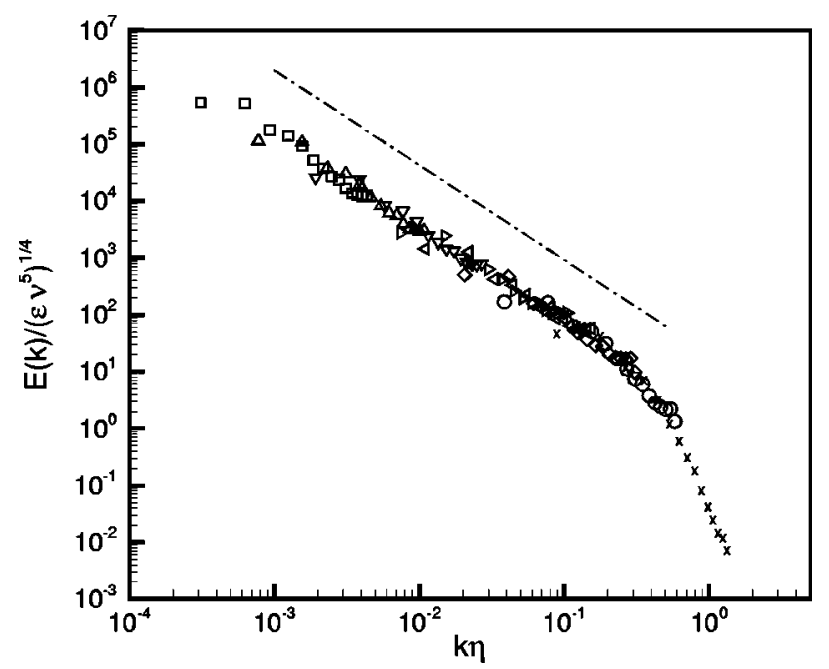

FIG. 7. Resolved flow energy spectra, LES (spiral). Cross: $\operatorname{Re}_{\lambda}=27$. Circle: $\operatorname{Re}_{\lambda}=52$. Diamond: $\operatorname{Re}_{\lambda}=84$. Left triangle: $\operatorname{Re}_{\lambda}=134$. Right triangle: $\operatorname{Re}_{\lambda}$ $=180$. Inverted triangle: $\operatorname{Re}_{\lambda}=444$. Triangle: $\operatorname{Re}_{\lambda}=810$. Square: $\operatorname{Re}_{\lambda}$ $=1540$. Dash-dotted line: slope $-5 / 3$.

$>500$ approximately, the SGSc model provides almost all of the dissipation. The subgrid energy dissipation ratio $\varepsilon_{\mathrm{SGS}} / \varepsilon$ (not plotted) showed similar behavior versus $\operatorname{Re}_{\lambda}$. Figure 6 also illustrates the effect of the basic SGS models on the LES for $\operatorname{Re}_{\lambda}$ smaller than 100. The $\operatorname{LES}(J=1.0)$ results show that the model begins to dissipate, when $\operatorname{Re}_{\lambda}$ increases above 27, just after full resolution is lost at $32^{3}$ resolution. This may indicate that the choice $J=1$ may be desirable for LES with the stretched-vortex SGS model for the purposes of LES at moderate $\mathrm{Re}_{\lambda}$, for resolutions at which the flow is underresolved.

The resolved velocity (energy) spectra scaled in the Kolmogorov form $E(k) /\left(\varepsilon \nu^{5}\right)^{1 / 4}$ vs $k \eta$ for the LES(spiral) results are shown in Fig. 7. Each spectrum is an average of four time frames over each of eight LES runs. The LES results appear to collapse reasonably onto a line with slope $-5 / 3$ in $\log -\log$ coordinates. The spectra for the LES $(J$ $=1)$ and $\operatorname{LES}(J=\infty)$ runs (not shown) were similar to those of Fig. 7. The resolved scalar spectra in Obukov-Corrsin scaling $E^{c}(k) \varepsilon^{3 / 4} /\left(\varepsilon^{c} \nu^{5 / 4}\right)$ are shown in Fig. 8 for the LES(spiral) runs. The envelope of all the spectra shown indicate a slope of $-5 / 3$ but there is a deviation from this at the high $k$ end of the resolved range for each separate LES, and the collapse is not as good as that seen for the corresponding energy spectra of Fig. 7.

\section{CONCLUDING REMARKS}

We have proposed and tested a subgrid model for the flux of a passive scalar in the large-eddy simulation of turbulent flows. The scalar subgrid flux is derived from the stretched-vortex subgrid-stress model by assuming that the principal contribution to the subgrid scalar flux is the convection of the local resolved field by the axisymmetric part of the velocity field of a model subgrid vortex. The local anisotropy of this process, as represented by the orientation of the subgrid vortex, produces a tensor-diffusion subgrid flux with coefficients whose magnitude depends on the

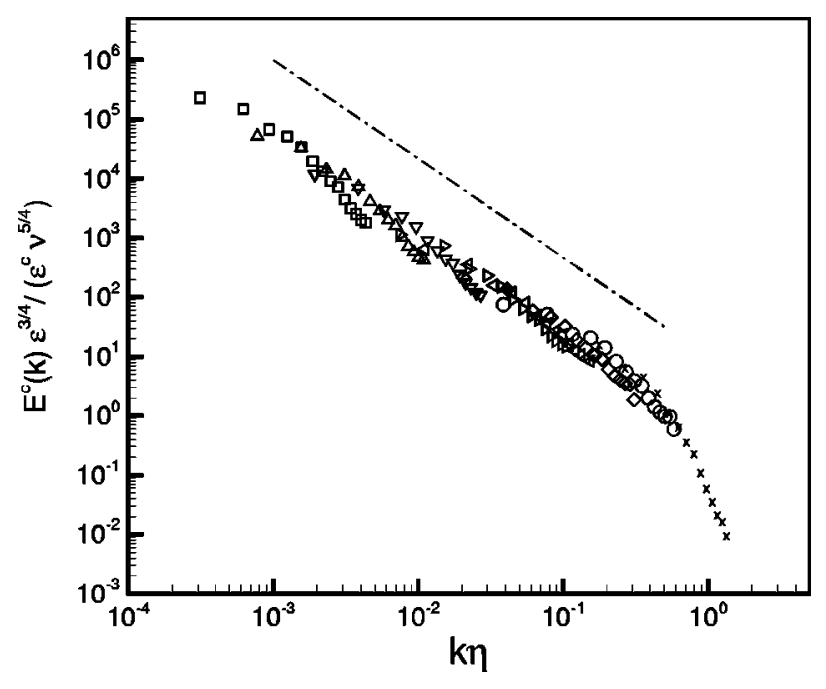

FIG. 8. Resolved flow scalar spectra, LES (spiral). For key see Fig. 7.

stretched-vortex estimate of the subgrid kinetic energy and the direction cosines of the vortex. Because values of these parameters are already known, calculation of the subgrid scalar flux requires only the additional determination of scalar gradients. The present form of the model is restricted to Schmidt numbers $\mathrm{Sc} \leqslant 1$, but could, with a more detailed model of the subgrid energy spectrum in the dissipation range than those used presently, and with additional assumptions concerning the subgrid contribution to the scalar variance, be extended to larger Sc. We have presently implemented the model for homogeneous isotropic turbulence but remark that because it is formulated in physical space, uses only local information, and does not assume the existence of homogeneous flow directions for the purposes of averaging, it can in principle be applied to the large-eddy simulation of nonhomogeneous flows.

Our main results are Eq. (24), which defines the subgrid scalar model, and Figs. 3 and 4 which best illustrate the model performance compared to detailed DNS (Ref. 4) of the statistics of a passive scalar with given mean gradient in homogeneous isotropic turbulence. The present large-eddy simulations indicate that at large $\operatorname{Re}_{\lambda}$, the dimensionless scalar variance and the ratio of the energy-dissipation time scale to the scalar-dissipation time scale become independent of $\operatorname{Re}_{\lambda}$, in agreement with standard scaling arguments. For the present test flow, consisting of passive scalar transport by forced isotropic turbulence with a preserved mean scalar gradient, the LES provides the numbers $c^{\prime 2} /\left(\alpha_{1} L_{\epsilon}\right)^{2} \approx 0.36$ and $\left(3 u^{\prime 2} / \varepsilon\right) /\left(c^{\prime 2} / \varepsilon^{c}\right) \approx 2.8$. The LES gives good collapse for the velocity spectrum in Kolmogorov scaling units, over a wide range of $\operatorname{Re}_{\lambda}$. The scalar spectra are not self similar, when plotted using Obukov-Corrsin scaling, at wave numbers near the resolved-scale cutoff. This may indicate that the present version of the scalar-flux model is somewhat overdissipative near the cutoff wave number. The model contains a single dimensionless free parameter, $\gamma$ in (24), which is presently set to unity. Future work will focus on the development of procedures for the dynamic calculation of $\gamma$. 


\section{ACKNOWLEDGMENT}

This work was supported in part by the National Science Foundation under Grant No. CTS-9978551.

${ }^{1} \mathrm{H}$. Tennekes and J. L. Lumley, A First Course in Turbulence (MIT Press, Cambridge, 1974).

${ }^{2} \mathrm{M}$. Lesieur and O. Métais, "New trends in large-eddy simulations of turbulence,” Annu. Rev. Fluid Mech. 28, 45 (1996).

${ }^{3}$ P. Moin, K. Squires, W. Cabot, and S. Lee, "A dynamic subgrid scale model for compressible turbulence an scalar transport," Phys. Fluids A 3, 2746 (1991).

${ }^{4}$ M. R. Overholt and S. B. Pope, "Direct numerical simulation of a passive scalar with imposed mean gradient in isotropic turbulence," Phys. Fluids 8, 3128 (1996).

${ }^{5}$ D. I. Pullin and P. G. Saffman, "Reynolds stresses and one-dimensional spectra for a vortex model of homogeneous anisotropic turbulence," Phys. Fluids 6, 1787 (1994).

${ }^{6}$ D. I. Pullin and P. G. Saffman, "Vortex dynamics in turbulence," Annu. Rev. Fluid Mech. 30, 31 (1998).
${ }^{7}$ A. Misra and D. I. Pullin, “'A vortex-based subgrid stress model for largeeddy simulation," Phys. Fluids 9, 2443 (1997).

${ }^{8}$ T. Voelkl, D. I. Pullin, and D. C. Chan, "A physical-space version of the the stretched-vortex subgrid-stress model for large-eddy simulation," Phys. Fluids 12, 1810 (2000).

${ }^{9}$ O. Métais and M. Lesieur, "Spectral large-eddy simulation of isotropic and stably stratified turbulence," J. Fluid Mech. 239, 157 (1992).

${ }^{10}$ T. S. Lundgren, "Strained spiral vortex model for turbulent fine structure," Phys. Fluids 25, 2193 (1982).

${ }^{11}$ G. R. Ruetsch and J. H. Ferziger, "Effects of small-scale structure on turbulent mixing," Center for Turbulence Research, Annual Research Briefs, 1997, pp. 213-235.

${ }^{12}$ D. K. Lilly, in Lectures Notes on Turbulence, edited by J. R. Herring and J. C. Mc Williams (World Scientific, Singapore, 1987), pp. 171-218.

${ }^{13}$ D. B. Spalding, "Concentration fluctuations in a round turbulent free jet," Chem. Eng. Sci. 26, 95 (1971).

${ }^{14}$ Z. Warhaft and J. L. Lumley, "An experimental study of the decay of temperature fluctuations in grid-generated turbulence," J. Fluid Mech. 88, 659 (1978). 